\title{
Anyons in a weakly interacting system
}

\author{
C. Weeks, G. Rosenberg, B. Seradjeh and M. Franz \\ Department of Physics and Astronomy, University of British Columbia, Vancouver, BC, Canada V6T $1 Z 1$
}

(Dated: June 30, 2018)

\begin{abstract}
In quantum theory, indistinguishable particles in three-dimensional space behave in only two distinct ways. Upon interchange, their wavefunction maps either to itself if they are bosons, or to minus itself if they are fermions. In two dimensions a more exotic possibility arises: upon exchange of two particles called "anyons" the wave function acquires phase $e^{i \theta} \neq \pm 1$. Such fractional exchange statistics are normally regarded as the hallmark of strong correlations. Here we describe a theoretical proposal for a system whose excitations are anyons with the exchange phase $\theta=\pi / 4$ and charge $-e / 2$, but, remarkably, can be built by filling a set of single-particle states of essentially noninteracting electrons. The system consists of an artificially structured type-II superconducting film adjacent to a $2 \mathrm{D}$ electron gas in the integer quantum Hall regime with unit filling fraction. The proposal rests on the observation that a vacancy in an otherwise periodic vortex lattice in the superconductor creates a bound state in the 2DEG with total charge $-e / 2$. A composite of this fractionally charged hole and the missing flux due to the vacancy behaves as an anyon. The proposed setup allows for manipulation of these anyons and could prove useful in various schemes for fault-tolerant topological quantum computation.
\end{abstract}

\section{INTRODUCTION}

Anyons and fractional charges appear in a variety of theoretical models involving electron or spin degrees of freedom in 2D [1, 2, 3, 4, 5, 6]. In all known cases, interactions between the fundamental degrees of freedom are of paramount importance for the emergence of the exotic excitations. For example, it is understood that the Coulomb interaction between electrons is crucial to stabilize the incompressible ground state in the fractional quantum Hall (FQH) liquids that support fractionalized excitations. One can say that such systems are strongly correlated in the sense that their many-body wavefunctions cannot be constructed from the singleparticle states of the constituent fundamental degrees of freedom.

The question thus arises whether anyons and fractionalization are inextricably connected with the phenomenon of strong correlations as defined above. There are compelling reasons to believe that this is in fact not so. In one dimension, Su, Schrieffer and Heeger 7] showed that a half of an electron can be bound to a domain wall in trans-polyacetylene modeled by a dimerized tight binding chain. Interactions play no role in this construction and the many-body wavefunction indeed can be built as a Slater determinant of single-particle electron states. In this case fractionalization is a many-body effect in that it is a cooperative phenomenon of a large number of electrons but it arises from the geometry of the system rather than strong correlations.

In three dimensions, Jackiw and Rebbi [8] argued that it is consistent to assign a half-integer fermion number to a monopole in the Yang-Mills gauge field coupled to relativistic Dirac fermions. In essence, this implies that a half-fermion is permanently bound to such a monopole. Again, interactions between fermions play no role in this construction and the half-fermion emerges from a manybody wavefunction that is a Slater determinant of single- particle states. It has been pointed out very recently [9] that a two-dimensional version of the physics underlying the above effect could be observable under certain conditions in graphene.

An appealing feature of weakly interacting systems is that their properties can be often understood from essentially exact calculations, which is rarely possible for strongly correlated systems in dimension greater than one. It is thus desirable to study weakly interacting systems with exotic excitations if there is a chance that they can be realized in a laboratory. Our proposal for such a system is depicted in Fig. 1(a). It consists of a type-II superconducting film grown on top of a semiconductor heterostructure (such as GaAs/AlGaAs) hosting a $2 \mathrm{D}$ electron gas. When a magnetic field is applied perpendicular to this planar device, an Abrikosov lattice of vortices forms in the superconductor. This results in a periodic modulation of the magnetic field in the $2 \mathrm{DEG}$ with one-half of a magnetic flux quantum $\Phi_{0}=h c / e$ per unit cell. Devices like this have in fact been fabricated previously [11, 12, 13] and studied theoretically [14, 15].

A key new aspect of our proposal is a periodic array of pinning sites imprinted on the superconductor. Pinning sites are regions of weakened superconductivity, which attract vortex cores and can be created in a variety of ways [16]. When the number of vortices in the sample equals the number of pinning sites, each pin is occupied by a vortex and a periodic vortex lattice arises. The Magnetic field strength at which this happens is called the matching field $B_{M}$. When the field is slightly below (above) $B_{M}$, vacancies (interstitials) appear in the vortex lattice as illustrated in Fig. 1(b). Such defects in the vortex lattice then lead to a localized deficit or surplus of magnetic flux quantized in the units of $\Phi_{0} / 2$ shown in Fig. 1(c).

Our principal claim, which we justify below by a general argument and a detailed computation, is this: When the lowest Landau level of the 2DEG is filled by spin- 
(a)

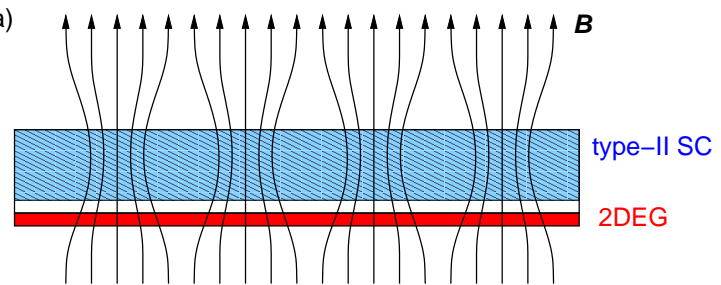

(b)

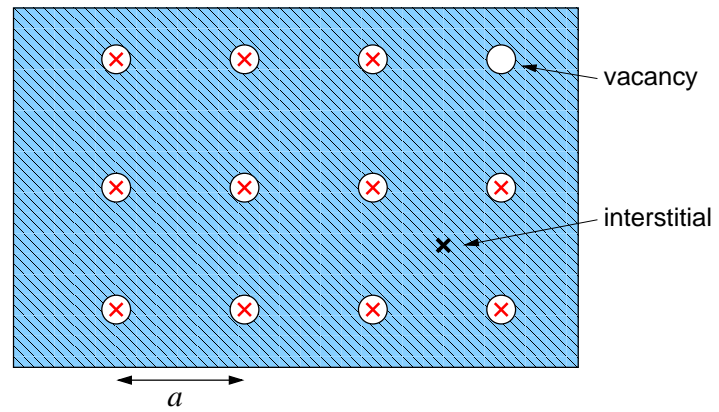

(c)

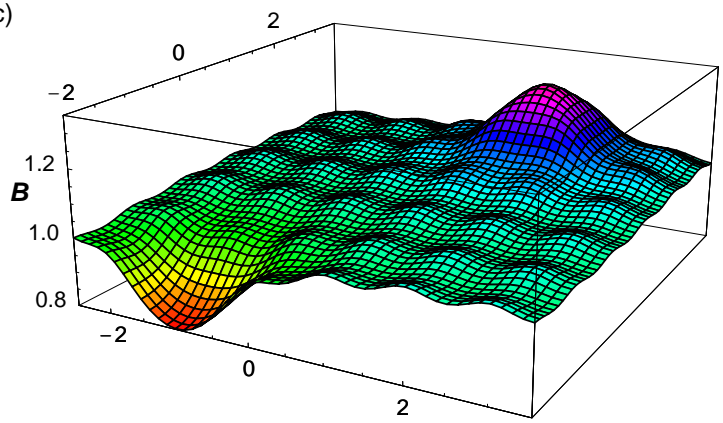

(d)

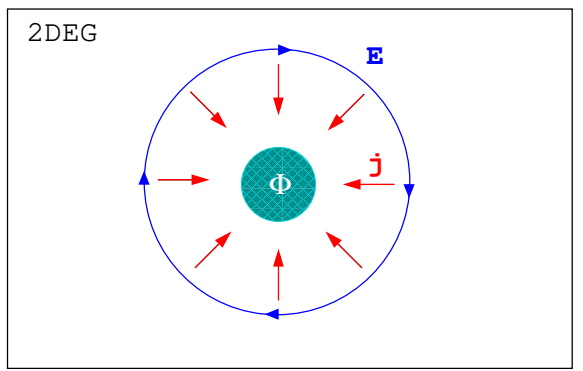

FIG. 1: (a) The schematic of the proposed device. Side view of the superconductor-2DEG heterostructure in an applied magnetic field B. (b) Top view. Open circles represent pinning sites in the superconductor while crosses denote vortices. Throughout this paper we consider, for simplicity, the square vortex lattice. All of our conclusions, however, remain valid for any arbitrary periodic Bravais lattice, including the most natural triangular case. (c) The magnetic field produced by a periodic square lattice of vortices with one vacancy (lower left corner) and one interstitial (upper right corner). The field distribution is calculated from a simple London model [10] with the penetration depth $\lambda=a$ and the vortex core cutoff $\xi=0.05 \lambda$. (d) Thought experiment: creating an interstitial by adiabatically threading flux through the system. polarized electrons, i.e. at filling fraction $\nu=1$, a defect in the vortex lattice binds fractional electric charge in the $2 \mathrm{DEG}$ with the exact value $-e / 2$ for a vacancy and $+e / 2$ for an interstitial. Such a defect constitutes a literal realization of Wilczek's model of an anyon as a bound state of magnetic flux and electric charge [17]. The exchange phase of this anyon $\pi / 4$.

The flux deficit/surplus can be, at least in principle, created, detected, and manipulated by the suite of scanning SQUID and Hall bar probes that have been developed over the years to image [18, 19, 20] and manipulate [21, 22] vortices in superconducting films and crystals. The proposed device could thus offer a unique opportunity to manipulate anyons with an unprecedented degree of control and advance the quest for a topologically protected quantum computer.

In order to execute a useful quantum computation, non-Abelian anyons are needed [4]. We remark that the device pictured in Fig. 11 could prove useful for the manipulation of such non-Abelian anyons if the 2DEG were put into the $\nu=\frac{5}{2} \mathrm{FQH}$ state, which is believed to be described by the Moore-Read "Pfaffian" state 23, 24, 25]. In that situation, vacancies and interstitials should bind non-Abelian anyons and the scanning SQUID probe could be used to locate these and possibly perform the braiding operations required to implement a fault tolerant quantum algorithm.

\section{ANYONS BOUND TO VACANCIES AND INTERSTITIALS: GENERAL ARGUMENTS}

So why does a vacancy bind fractional charge? The answer can be given at several levels of sophistication and is essentially implicit in the body of work on the FQH effect. The simplest way to see that this is true is to recall that electrons in the quantum Hall state at $\nu=1$ form an incompressible fluid. This means that in the ground state the electron charge density $\rho(\mathbf{r})$ tracks the magnetic field strength $B(\mathbf{r})$ in accordance with the defining relation

$$
\nu=\frac{N_{e}}{N_{\Phi}}=\frac{\rho \Phi_{0}}{e B},
$$

where $N_{e}$ and $N_{\Phi}$ denote the total number of electrons and flux quanta in the sample, respectively. In an incompressible state the above relation remains valid, to a good approximation, even for a spatially varying magnetic field, as long as the variation is weak. In our device, a slowly varying field occurs in the limit when the magnetic penetration depth $\lambda \gg a, a$ being the intervortex distance. In this limit, one obtains

$$
\rho(\mathbf{r}) \simeq \nu e \frac{B(\mathbf{r})}{\Phi_{0}} .
$$

This relation, which becomes exact when integrated over the region containing the vacancy, implies that at $\nu=1$ 
a deficit of one half flux quantum produces a deficit of one half of an electron in the 2DEG.

To see more clearly how the charge deficit/surplus comes about we may employ a version of Laughlin's argument [26] similar to that used by Halperin to deduce the existence of edge states in integer quantum Hall liquids [27]. The idea is to start from a perfect vortex lattice and imagine creating an interstitial by adiabatically tuning the flux of the extra vortex from zero to $\Phi_{0} / 2$. We again consider the limit $\lambda \gg a$.

According to Faraday's law, $\nabla \times \mathbf{E}=-(1 / c)(\partial \mathbf{B} / \partial t)$, the time dependent magnetic field associated with introducing the extra vortex induces an electric field with concentric field lines as shown in Fig. 1(d). In the quantum Hall liquid such an electric field produces current strictly perpendicular to the field,

$$
\mathbf{j}=\sigma_{x y}(\mathbf{E} \times \hat{z}),
$$

where $\sigma_{x y}=\nu e^{2} / h$ is the quantized Hall conductance. Using the continuity equation for the electric charge, one can integrate this current to find the rate of change of the total charge $Q$ in the region bounded by an arbitrary closed contour $\mathcal{C}$ enclosing the flux,

$$
\frac{d Q}{d t}=-\oint_{\mathcal{C}} d l \mathbf{n} \cdot \mathbf{j}=-\sigma_{x y} \oint_{\mathcal{C}} d \mathbf{l} \cdot \mathbf{E},
$$

where $\mathbf{n}$ is a unit vector normal to the contour. Using Stokes theorem, the last integral can then be writen as an integral of $\nabla \times \mathbf{E}$ over an area bounded by $\mathcal{C}$. This we can evaluate with help of Faraday's law to obtain $d Q / d t=\left(\sigma_{x y} / c\right) d \Phi / d t$. It follows that the charge $\delta Q$ accumulated inside $\mathcal{C}$ during the process of adiabatic flux insertion is

$$
\delta Q=e \nu \frac{\delta \Phi}{\Phi_{0}} .
$$

For $\nu=1$ and $\delta \Phi= \pm \Phi_{0} / 2$ the accumulated charge is $\pm e / 2$. It is to be noted that this argument depends only on the fundamental property (3) and the above result (5) should thus be valid unconditionally as long as the 2DEG remains in the quantum Hall plateau.

Vacancies and interstitials, being bound states of charge and flux, $\left(-e / 2,-\Phi_{0} / 2\right)$ and $\left(e / 2, \Phi_{0} / 2\right)$ respectively, acquire nontrivial Aharonov-Bohm phases upon adiabatic exchange, and can thus be thought of as anyons. The standard textbook counting procedure [28], according to which a charge $q$ taken around a flux $\Phi$ acquires a phase $2 \pi(q / e)\left(\Phi / \Phi_{0}\right)$, would imply a "semionic" exchange phase $\theta=\pi / 2$ for both vacancies and interstitials, and a mutual exchange phase $\theta_{M}=-\pi / 2$. This conclusion, however, is incorrect for the following reason. The charge $\pm e / 2$ is built up from fermionic degrees of freedom and thus has attached to it half of an electron spin, or more precisely, half of the electron statistical phase, which needs to be taken into account when computing the exchange phase of the composite object. The simplest way to arrive at the correct result is to consider fusion rules for anyons. The relevant rule [28] states that the exchange phase $\Theta$ of a particle formed by combining $n$ identical anyons with exchange phase $\theta$ is $\Theta=n^{2} \theta$.

Consider bringing together two interstitials. The resulting object is $\left(e, \Phi_{0}\right)$, a bound state of an electron and a full flux quantum. The exchange phase of this object is $\pi+2 \pi=3 \pi$, the first term reflecting the intrinsic electron exchange phase and the second the Aharonov-Bohm phase. But a $3 \pi$ phase is equivalent to $\pi$, i.e. the bound state is a fermion. The above fusion rule with $n=2$ thus determines the exchange phase of an interstitial to be $\theta=\pi / 4$. A similar argument leads to the identical result for a vacancy. These results will be confirmed below by explicit model calculations.

\section{CONTINUUM MODEL}

We first consider a very simple continuum model, which is exactly soluble and captures the essence of the effect discussed above. Consider the following 2dimensional electron Hamiltonian,

$$
\mathcal{H}=\frac{1}{2 m_{e}}\left(\mathbf{p}-\frac{e}{c} \mathbf{A}\right)^{2},
$$

with $m_{e}$ the electron mass, $\mathbf{p}$ the momentum operator in the $x-y$ plane and $\mathbf{A}=\mathbf{A}_{0}+\delta \mathbf{A}$. Here $\mathbf{A}_{0}=\frac{1}{2} B_{0}(\mathbf{r} \times \hat{z})$ represents a uniform field in the $\hat{z}$ direction and

$$
\delta \mathbf{A}=\frac{\eta \Phi_{0}}{2 \pi r^{2}}(\mathbf{r} \times \hat{z}) .
$$

The total field seen by an electron is then

$$
\mathbf{B}(\mathbf{r})=\nabla \times \mathbf{A}=\hat{z} B_{0}+\hat{z} \eta \Phi_{0} \delta(\mathbf{r}) .
$$

The $\delta$-function serves as a crude representation of the flux added by an interstitial $\left(\eta=\frac{1}{2}\right)$ or removed by a vacancy $\left(\eta=-\frac{1}{2}\right)$ in the vortex lattice.

It is straightforward to find the single particle eigenstates $\psi_{k m}(\mathbf{r})$ of $\mathcal{H}$ for arbitrary real $\eta$ by working in the polar coordinate basis. They are labeled by the principal quantum number $k=0,1,2, \ldots$ and an integer angular momentum $m$. The allowed energy levels read

$$
\epsilon_{k m}=\frac{1}{2} \hbar \omega_{c}[2 k+1+|m-\eta|-(m-\eta)],
$$

where $\omega_{c}=e B_{0} / m_{e} c$ is the cyclotron frequency. The spectrum (8) is depicted in Fig. 2(a). For non-integer $\eta$, in addition to the usual Landau levels, there also exist bound states associated with the extra flux.

The eigenstates $\psi_{0 m}$ in the lowest Landau level have a simple form

$$
\psi_{0 m}(z)=A_{m}|z|^{-\eta} z^{m} e^{-|z|^{2} / 4},
$$

where $z=(x+i y) / \ell_{B}$ is the dimensionless complex coordinate, $A_{m}=\left[\ell_{B}^{2} 2 \pi 2^{m-\eta} \Gamma(1+m-\eta)\right]^{-1 / 2}$ is the normalization constant and $\ell_{B}=\sqrt{\hbar c / e B}$ the magnetic length. 

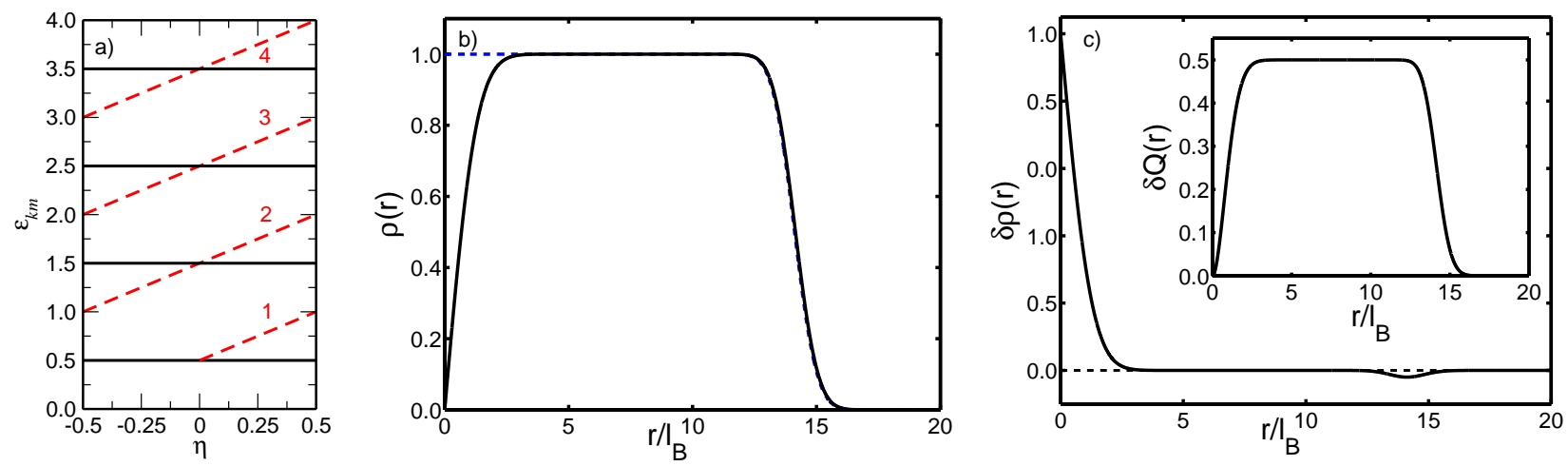

FIG. 2: (a) The energy levels (in units of $\hbar \omega_{c}$ ) of Hamiltonian (6) as a function of fractional flux $\eta$. The numerals denote degeneracy of bound state levels represented by dashed lines. Degeneracy of the extended states (solid lines) is proportional to the area of the system. (b) Charge density $\rho(r)$, in units of $e / 2 \pi \ell_{B}^{2}$, for $N=100$ as a function of distance $r$ from the origin. Solid line represents density $\rho_{0}(r)$ for uniform magnetic field, dashed line has a flux tube carrying $-\Phi_{0} / 2$ at the origin. (c) The excess charge density $\delta \rho(r)=\rho_{0}(r)-\rho(r)$. Inset shows the accumulated excess charge $\delta Q(r)=2 \pi \int_{0}^{r} r^{\prime} d r^{\prime} \delta \rho\left(r^{\prime}\right)$ in units of $e$.

If we fill the lowest Landau level with electrons, then the many-body wavefunction can be constructed as a Slater determinant of $\psi_{0 m}\left(z_{i}\right)$, where $z_{i}$ is a complex coordinate of the $i$-th electron. The usual simplifications using the properties of Vandermonde determinants then give

$$
\Psi\left(\left\{z_{i}\right\}\right)=\mathcal{N} \prod_{i}\left|z_{i}\right|^{-\eta} \prod_{i<j}\left(z_{i}-z_{j}\right) e^{-\sum_{i}\left|z_{i}\right|^{2} / 4} .
$$

We are interested in the charge density implied by the above many-body wavefunction, which is given as the expectation value $\rho(\mathbf{r})=\left\langle\Psi_{0}|\hat{\rho}| \Psi_{0}\right\rangle$ of the electron density operator $\hat{\rho}=e \sum_{i} \delta\left(\mathbf{r}-\mathbf{r}_{i}\right)$. For a droplet composed of $N$ electrons occupying the $N$ lowest angular momentum states we obtain

$$
\rho(\mathbf{r})=e \sum_{m=0}^{N-1}\left|\psi_{0 m}(\mathbf{r})\right|^{2} .
$$

The charge density obtained by numerically evaluating Eq. (11) is displayed in Fig. 2, both for a uniform field $(\eta=0)$ and the vacancy $\left(\eta=-\frac{1}{2}\right)$ placed at the origin. We observe that the inclusion of a vacancy leads to suppression of the charge density near the origin, the excess charge being deposited at the outer edge of the system. The integrated charge deficit $\delta Q(r)$ quickly saturates at $e / 2$, confirming the conclusion reached above by general argument. The vacancy in this model indeed binds half of an electron.

From the many-body wavefunction (10) one can obtain the charge and the statistical angle associated with a vacancy by the standard calculation [2] of the Berry phase. To compute the Berry phase it is convenient to perform a gauge transformation

$$
\delta \mathbf{A} \rightarrow \delta \mathbf{A}-\frac{\eta \Phi_{0}}{2 \pi} \nabla \varphi
$$

where $z=|z| e^{i \varphi}$. The resulting vector potential can be seen to vanish everywhere, except for a cut along the positive $x$ axis. The effect of the gauge transformation is to multiply the eigenstates (9) by $e^{-i \eta \varphi}$ which has the effect of replacing $|z|^{-\eta}$ by $z^{-\eta}$. In this "string gauge" the entire phase information is encoded in the wavefunction. The many-body wavefunction for a vacancy $\left(\nu=-\frac{1}{2}\right)$ located at the complex coordinate $w$ can thus be written as

$$
\Psi_{w}\left(\left\{z_{i}\right\}\right)=\mathcal{N}_{w} \prod_{i}\left(z_{i}-w\right)^{1 / 2} \Psi_{0}\left(\left\{z_{i}\right\}\right),
$$

where $\Psi_{0}\left(\left\{z_{i}\right\}\right)=\prod_{i<j}\left(z_{i}-z_{j}\right) e^{-\sum_{i}\left|z_{i}\right|^{2} / 4}$.

If we now adiabatically carry the vacancy along a closed contour $\mathcal{C}$ the wavefunction acquires a purely geometric phase

$$
\gamma(\mathcal{C})=i \oint_{\mathcal{C}} d w\left\langle\Psi_{w} \mid \frac{\partial}{\partial w} \Psi_{w}\right\rangle
$$

Calculation of $\gamma(\mathcal{C})$ proceeds exactly as in Ref. [2] and yields

$$
\gamma(\mathcal{C})=-\pi \frac{\Phi}{\Phi_{0}}
$$

where $\Phi$ is the total magnetic flux enclosed by the contour $\mathcal{C}$. This result coincides with the Aharonov-Bohm phase acquired by a charge $-e / 2$ carried along a trajectory enclosing flux $\Phi$, confirming once again our earlier result for the electric charge of the vacancy.

For two vacancies, located at $w_{a}$ and $w_{b}$ the manybody wavefunction reads

$$
\Psi_{w_{a} w_{b}}=\mathcal{N}_{w_{a} w_{b}} \prod_{i}\left(z_{i}-w_{a}\right)^{1 / 2}\left(z_{i}-w_{b}\right)^{1 / 2} \Psi_{0} .
$$

If we now carry $w_{a}$ along a contour $\mathcal{C}$ that encloses $w_{b}$, the latter held static, then Eq. (14) gives the Berry phase

$$
\gamma(\mathcal{C})=-\pi\left(\frac{\Phi}{\Phi_{0}}-\frac{1}{2}\right)
$$


The first term reflects the Aharonov-Bohm phase as before. Since the encircling operation can be thought of as two consecutive exchanges we must interpret the second term as twice the exchange phase of the vacancy, $\theta=\pi / 4$.

\section{LATTICE MODEL}

Thus far we have ignored the periodic variation of the magnetic field induced by the vortex lattice. A question thus arises whether this could affect the emergence of anyons in realistic systems. To see that this is not the case we now consider a model which describes the limit of strong field variation and also includes the effect of a Zeeman interaction.

If the effective Zeeman coupling in a 2DEG is sufficiently strong, then, in addition to a quasi-periodic vector potential, the electrons also feel a periodic scalar potential. This effect becomes important in diluted magnetic semiconductors, such as $\mathrm{Ga}_{1-x} \mathrm{Mn}_{x} \mathrm{As}$ or $\mathrm{Cd}_{1-x} \mathrm{Mn}_{x} \mathrm{Te}$, where the effective gyro-magnetic ratio can be of the order $\sim 10^{2}-10^{3}$. As argued in Ref. [15], in the limit $\lambda \ll a$, electrons in a 2DEG become almost localized near the maxima of the Zeeman field and can be described by a tight binding model with one-half magnetic flux quantum per unit cell.

This motivates our consideration of a tight binding Hamiltonian

$$
\mathcal{H}=-\sum_{i j}\left(t_{i j} e^{i \theta_{i j}} c_{j}^{\dagger} c_{i}+\text { h.c. }\right)+\sum_{i} \epsilon_{i} c_{i}^{\dagger} c_{i},
$$

where $c_{i}^{\dagger}$ creates an electron on a site $\mathbf{r}_{i}$ of a square lattice, and $t_{i j}$ and $\epsilon_{i}$ are real hopping amplitudes and onsite energies, respectively. The effect of magnetic field is included by the Peierls phase factors

$$
\theta_{i j}=\frac{2 \pi}{\Phi_{0}} \int_{\mathbf{r}_{i}}^{\mathbf{r}_{j}} \mathbf{A} \cdot \mathbf{d} \mathbf{l}
$$

In the following we consider the case of nearest and nextnearest neighbor hopping amplitudes $t$ and $t^{\prime}$ respectively, but note the addition of longer range hoppings does not affect our results.

In the absence of defects we have $\epsilon_{i}=\epsilon=$ const and the magnetic flux through each plaquette is $\Phi_{0} / 2$. The spectrum of the Hamiltonian (18) consists of two branches, reflecting the fact that the unit cell now contains two lattice sites. In the Landau gauge, $\mathbf{A}=B_{0} \hat{y} x$ with $B_{0}=\Phi_{0} / 2 a^{2}$, the allowed energies read

$$
E_{\mathbf{k}}=\epsilon \pm 2 t\left[\cos ^{2} k_{x}+\cos ^{2} k_{y}+4 \gamma^{2} \sin ^{2} k_{x} \sin ^{2} k_{y}\right]^{1 / 2}
$$

where $\mathbf{k}$ is a wavevector drawn from the first magnetic Brillouin zone, $\gamma=t^{\prime} / t$, and we have set $a=1$. For $t^{\prime}=0$ the spectrum exhibits two Dirac points at $( \pm \pi / 2, \pi / 2)$. Inclusion of next-nearest neighbor hopping breaks the time reversal symmetry and produces a gap at these two points, as illustrated in Fig. 3(a). We are interested in the half-filled case with the lower band filled and the upper band empty. This situation is analogous to the $\nu=1$ continuum case with Hall conductance $\sigma_{x y}=e^{2} / h$ discussed previously.

A vacancy in the vortex lattice removes flux $\Phi_{0} / 2$ from the plaquettes adjacent to the affected site, modifies its on-site energy $\epsilon_{i}$ and possibly also those of the nearby sites. While the total flux removed is exactly $\Phi_{0} / 2$, the change of the on-site energies $\delta \epsilon_{i}$ depends on the details of the underlying microscopic model for the Zeeman interaction. We find that, as expected by the general argument, the total charge deficit associated with a vacancy depends critically on the total flux removed but is independent of $\delta \epsilon_{i}$ or the details of the flux distribution.

We have solved the model specified by Hamiltonian (18) by exact numerical diagonalization for lattice sizes up to $50 \times 50$ sites. We have considered various configurations of $\delta \epsilon_{i}$. In all cases we found that the total charge induced by an interstitial/vacancy is $\pm e / 2$, to within machine accuracy. This result continues to hold even in the presence of randomness in the on-site energies, as long as the randomness is weak compared to the gap. This is illustrated in Fig. 3(b-f).

\section{SUMMARY AND CONCLUSIONS}

Our foregoing discussion shows how two simple and well understood systems, a type-II superconductor and a 2-dimensional electron gas in the integer quantum Hall regime, can exhibit some rather unusual properties when brought into close proximity. These include nontrivial exchange statistics and charge fractionalization, properties that are normally thought of as hallmarks of strong correlation physics. In our proposed device these phenomena occur in what must be characterized as a weakly correlated system: the many-body wavefunctions of electrons in both subsystems are conventional Slater determinants of single-particle states.

There are two key ingredients that give rise to the above unusual properties: (i) precise flux quantization in a type-II superconductor and, (ii) incompressibility of the 2DEG at integer filling $\nu$. The device displayed in Fig. 1 is designed to project a precisely quantized deficit or surplus of magnetic flux onto the 2DEG, which responds by adjusting its many-body wavefunction to produce a localized deficit or surplus of electric charge with precise value $e / 2$. This phenomenon can be regarded as a consequence of gauge invariance and is therefore robust against any weak but otherwise arbitrary perturbation imposed on the system.

A question that we must ask is to what extent are the fractional charges associated with the vacancies and interstitials legitimate quasiparticle excitations of the system. Surely, from the point of view of a 2DEG at integer filling, such objects are not natural excitations. Indeed, in our device they occur in response to the local change 


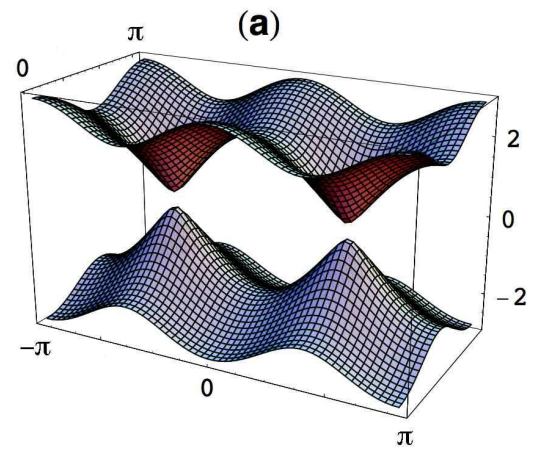

(d)

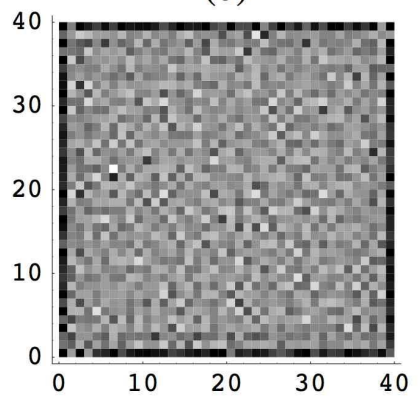

(b)

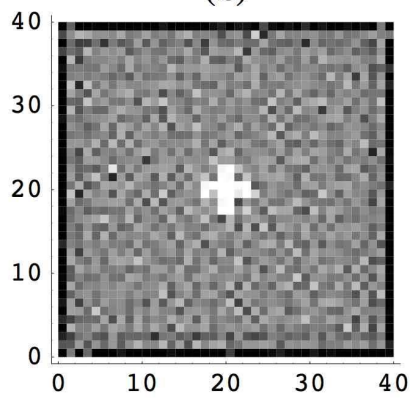

(e)

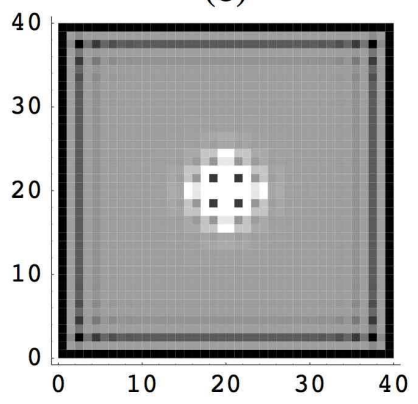

(c)

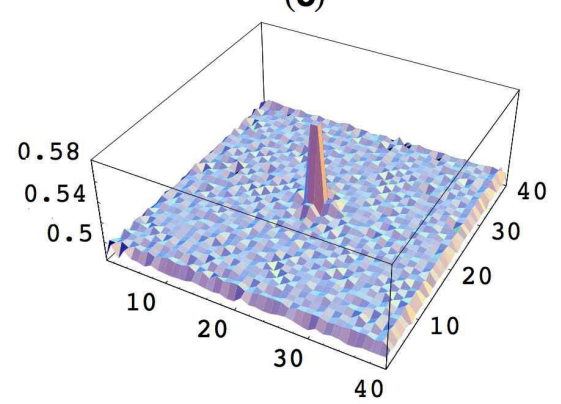

(f)

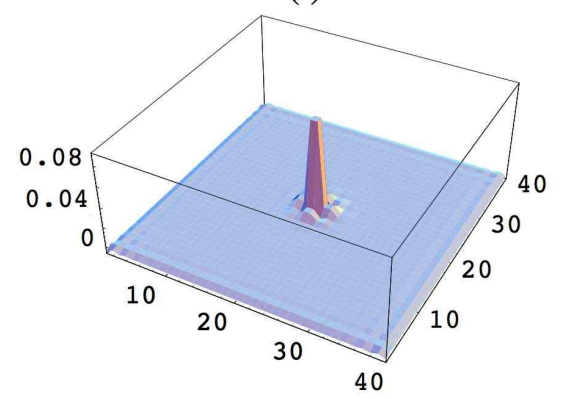

FIG. 3: a) Band structure of the tight binding model on a square lattice with one half flux quantum threading each plaquette and $t^{\prime}=0.05 t$. b,c) Charge density $\rho$ at half filling with random on-site energies (Gaussian distribution with $\Delta \epsilon=0.05 t^{\prime}$ ) and a full flux quantum modeling the interstitial piercing the central plaquette. d) Charge density $\rho_{0}$ with the same realization of randomness but without the extra flux. e,f) The charge density $\delta \rho=\rho-\rho_{0}$ induced by the interstitial. The induced charge $\delta Q$ integrates to $e / 2$ to within machine accuracy.

in the magnetic flux, which is external to the 2DEG. The key point is that fractional charges bound to defects are excitations of the entire system in the same sense as fractional charges bound to domain walls are excitations of a dimerized polyacetylene chain 7 ].

Fractional charges should be relatively easy to detect in a transport experiment in the regime where the longitudinal conductivity of the 2DEG is furnished solely by the $e / 2$ defects. Fractional statistics, on the other hand, will be more difficult to establish since this requires an interference experiment; in essence we need a defect to quantum-delocalize and interfere with itself after encircling another defect. As noted recently, non-trivial statistics might be easier to detect in the $\nu=\frac{5}{2}$ non-Abelian case [29, 30].

Aside from demonstrating the possibility of unconventional phenomena in a weakly interacting system, our proposed device could allow for the manipulation of anyons in the context of quantum information processing. Indeed, some schemes for fault tolerant topological quan- tum computation [4, 5, 6] involve execution of braiding and fusion operations on anyons. In the present setup these could be facilitated by the fact that our anyons are permanently bound to physical magnetic fluxes and well developed techniques exist to detect and manipulate these. As already remarked above if the 2DEG in our device were tuned into the $\nu=\frac{5}{2}$ Moore-Read Pfaffian state then a defect in the vortex lattice would bind a quasiparticle with non-Abelian exchange statistics. Having a "handle" on such a quasiparticle in the form of the attached magnetic flux could prove useful for realizing the requisite braiding and fusion operations.

Acknowledgments - The authors are indebted to M. Berciu and A. Vishwanath for some key suggestions and wish to thank I. Affleck, D. Fisher, D. Haldane, C. Kallin, A. Kitaev, K. Shtengel, D. Scalapino and Z. Tesanovic for stimulating discussions and correspondence. The work reported here was supported by NSERC, CIAR and the Killam Foundation.
[1] Laughlin, R.B. Anomalous Quantum Hall Effect: An Incompressible Quantum Fluid with Fractionally Charged Excitations, Phys. Rev. Lett. 50, 1395-1399 (1983).

[2] Arovas, D., Schrieffer, J.R. and Wilzcek, F. Fractional
Statistics and the Quantum Hall Effect, Phys. Rev. Lett. 53, 722-723 (1984).

[3] Wen, X.G. Mean-field theory of spin-liquid states with finite energy gap and topological orders, Phys. Rev. Lett. 
44, 2664-2672 (1991).

[4] Kitaev, A. Yu. Fault-tolerant quantum computation by anyons, Ann. Phys. 303, 2-30 (2003).

[5] Freedman, M., Nayak, C., Shtengel, K., Walker, K. and Wang, Z. A class of P,T-invariant topological phases of interacting electrons, Ann. Phys. 310, 428-492 (2004).

[6] Kitaev, A. Yu. Anyons in an exactly solved model and beyond, Ann. Phys. 321, 2-111 (2006).

[7] Su, W.P., Schrieffer, J.R. and Heeger, A.J. Solitons in Polyacetylene, Phys. Rev. Lett. 42, 1698-1701 (1979).

[8] Jackiw, R. and Rebbi, C. Solitons with fermion number $\frac{1}{2}$, Phys. Rev. D 13, 3398-3409 (1976).

[9] Hou, C.-Y., Chamon, C. and Mudry C. Electron fractionalization in two-dimensional graphene-like structures (unpublished, preprint cond-mat/0609740).

[10] See e.g. M. Tinkham, Introduction to Superconductivity (McGraw-Hill, New York, 1996)

[11] Bending, S.J., von Klitzing, K. and Ploog K. Weak localization in a distribution of magnetic flux tubes, Phys. Rev. Lett. 65, 1060-1063 (1990).

[12] Geim, A.K., Bending, S.J., and Grigorieva I.V. Asymmetric scattering and diffraction of two-dimensional electrons at quantized tubes of magnetic flux, Phys. Rev. Lett. 69, 2252-2255 (1992).

[13] Danckwerts, M., Goi, A.R., Thomsen, C., Eberl, K. and Rojo, A.G. Enhanced Vortex Damping by Eddy Currents in Superconductor-Semiconductor Hybrids, Phys. Rev. Lett. 84, 3702-3705 (2000).

[14] Rammer, J. and Shelankov, A.L. Weak localization in inhomogeneous magnetic fields, Phys. Rev. B 36, 31353146 (1987).

[15] Berciu, M., Rappoport, T.G. and Janko, B. Manipulating spin and charge in magnetic semiconductors using superconducting vortices, Nature 435, 71-75 (2005).

[16] See e.g. U. Welp, U., Xiao, Z.L., Novosad, V. and VlaskoVlasov, V.K. Commensurability and strong vortex pinning in nanopatterned Nb films, Phys. Rev. B 71, 014505 (2005) and references 1-11 therein.

[17] Wilczek, F. Quantum mechanics of fractional-spin particles, Phys. Rev. Lett. 49, 957-959 (1982).
[18] Kirtley, J.R. et al. Direct Imaging of Integer and HalfInteger Josephson Vortices in High- $T_{c}$ Grain Boundaries, Phys. Rev. Lett. 76, 1336-1339 (1996).

[19] Tafuri, F., Kirtley, J.R., Medaglia, P.G., Orgiani, P. and Balestrino, G. Magnetic Imaging of Pearl Vortices in Artificially Layered $\left(\mathrm{Ba}_{0.9} \mathrm{Nd}_{0.1} \mathrm{CuO}_{2+x}\right)_{m} /\left(\mathrm{CaCuO}_{2}\right)_{n}$ Systems, Phys. Rev. Lett. 92, 157006 (2004).

[20] Bluhm, H., Sebastian, S.E., Guikema, J.W., Fisher, I.R. and Moler, K.A. Scanning Hall probe imaging of $\mathrm{ErNi}_{2} \mathrm{~B}_{2} \mathrm{C}$, Phys. Rev. B 73, 014514 (2006).

[21] Gardner, B.W. et al. Manipulation of single vortices in $\mathrm{YBa}_{2} \mathrm{Cu}_{3} \mathrm{O}_{6.354}$ with a locally applied magnetic field, Appl. Phys. Lett. 80, 1010 (2002).

[22] Gardner, B.W. et al. Vortex-antivortex annihilation in underdoped $\mathrm{YBa}_{2} \mathrm{Cu}_{3} \mathrm{O}_{6.354}$, Physica C 388-389, 725726 (2003).

[23] Moore, G. and Read, N. Nonabelions in the fractional quantum Hall effect, Nucl. Phys. B 360, 362-396 (1991).

[24] Nayak, C. and Wilczek, F. 2n-quasihole states realize $2^{n-1}$-dimensional spinor braiding statistics in paired quantum Hall states, Nucl. Phys. B 479, 529-553 (1996).

[25] Read, N. and Green, D. Paired states of fermions in two dimensions with breaking of parity and time-reversal symmetries and the fractional quantum Hall effect, Phys. Rev. B 61, 10267-10297 (2000).

[26] Laughlin, R.B. Quantized Hall conductivity in two dimensions, Phys. Rev. Lett. 23, 5632-5633 (1981).

[27] Halperin, B.I. Quantized Hall conductance, currentcarrying edge states, and the existence of extended states in a two-dimensional disordered potential, Phys. Rev. B 25, 2185 - 2190 (1982).

[28] Wilczek, F. Fractional statistics and anyon superconductivity (World Scientific, Singapore, 1990).

[29] Das Sarma, S., Freedman, M. and Nayak C. Topologically Protected Qubits from a Possible Non-Abelian Fractional Quantum Hall State, Phys. Rev. Lett. 94, 166802 (2005).

[30] Bonderson, P., Kitaev, A. and Shtengel, K. Detecting Non-Abelian Statistics in the $\nu=5 / 2$ Fractional Quantum Hall State, Phys. Rev. Lett. 96, 016803 (2006). 